\title{
Investigation of the influence of plasma source power on the properties of magnetron sputtered $\mathrm{Ta}_{2} \mathrm{O}_{5}$ thin films
}

\author{
Manuel Bärtschi ${ }^{1,3}$, Daniel Schachtler ${ }^{l}$, Silvia Schwyn-Thöny², Thomas Südmeyer ${ }^{3}$ and Roelene Botha ${ }^{1}$ \\ ${ }^{I}$ RhySearch, the Research and Innovation Center in the Alpine Rhine Valley, Werdenbergstrasse 4, 9471 Buchs SG, Switzerland \\ ${ }^{2}$ Evatec Ltd., Hauptstrasse 1a, 9477 Trübbach, Switzerland \\ ${ }^{3}$ Laboratoire Temps-Fréquence, Université de Neuchâtel, 2000 Neuchâtel, Switzerland
}

\begin{abstract}
To enable the production of sophisticated optical interference coating designs, coatings with very low absorption and stray light losses and excellent layer thickness deposition accuracy are required. The selection and optimization of suitable coating materials and deposition processes are consequently essential. This study investigated the influence of the plasma source power on the optical properties, layer uniformity and stress, scattered light behavior and optical losses of magnetron sputtered $\mathrm{Ta}_{2} \mathrm{O}_{5}$ thin films.
\end{abstract}

\section{Introduction}

In the field of optical thin-film technology, it is essential to characterize the properties of the dielectric material and the coating equipment used, to meet the requirements of the ever-increasing range of applications. Tantalum pentoxide $\left(\mathrm{Ta}_{2} \mathrm{O}_{5}\right)$ is a widely used material for optical interference coatings due to its high refractive index $[1,2]$. Likewise, magnetron sputtering is a technology of choice for the mass production of optical coatings, offering high deposition rates and high throughputs [3]. This work consequently investigates the properties of $\mathrm{Ta}_{2} \mathrm{O}_{5}$ thin films and their production using the latest generation magnetron sputtering equipment.

\section{Experimental setups}

During the experiments, the Clusterline200 BPME, a magnetron sputter tool from Evatec AG, was used (Figure 1). It allows coating 15 substrates with a diameter of $20 \mathrm{~cm}$ simultaneously. Besides load locks for loading and unloading the substrates, an aligner, a handler and a variety of other secondary equipment, the main part of the system is the coating vacuum chamber. It consists of a rotating table with 15 substrate carriers which rotate as well. Three plasma sources are located on the top of the coating vacuum chamber. For this work, one magnetron sputtering source with a tantalum target, one with a silicon target and one as an additional argon-oxide plasma source (PSC) was used. The magnetron sputter source is powered by a DC-generator and uses a rotating magnetic system to obtain an optimal distribution and enable the mixture of oxygen and argon to be adjusted.

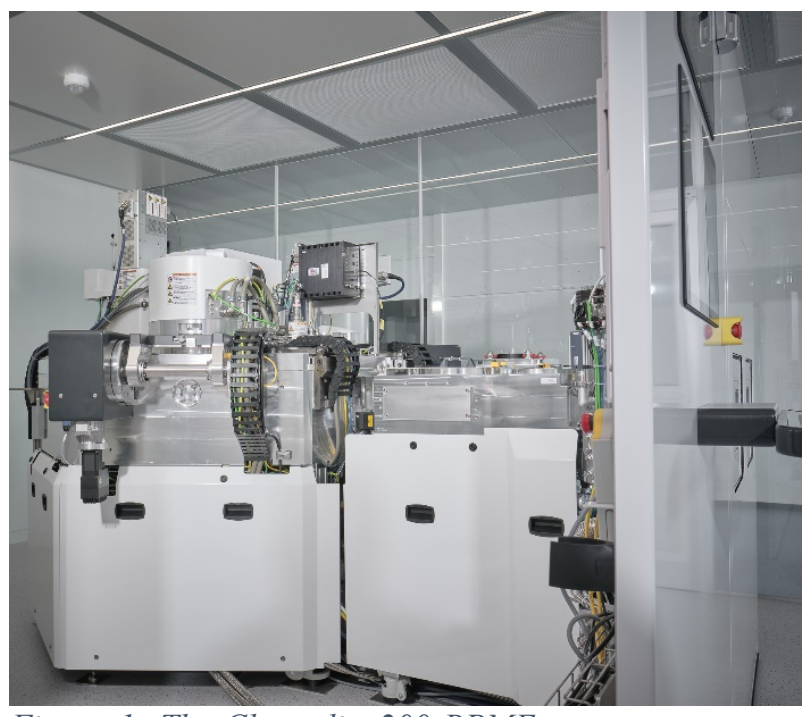

Figure 1: The Clusterline200 BPME magnetron sputter tool.

The PSC works as a capacitively coupled RF-plasma source and can be operated with argon for increased energy during the coating process with oxygen to oxidize the coated layer (Figure 2). Various improvements in material properties have been achieved using the PSC for depositing silicon dioxide $\left(\mathrm{SiO}_{2}\right)$ and hafnium dioxide $\left(\mathrm{HfO}_{2}\right)$ [4]. The coating process is monitored by an optical broadband monitoring system (GSM) to calculate the actual layer thickness at a specific time during the deposition process by measuring the changing reflection from a monitoring sample coated together with the production substrates. By comparing the reflection spectrum with the expected spectrum for the specific growing layer, the optimal deposition time can be used to

\footnotetext{
${ }^{*}$ Corresponding author: manuel.baertschi@rhysearch.ch
} 
ensure the best possible accordance of the measured layer spectrum with the desired design.

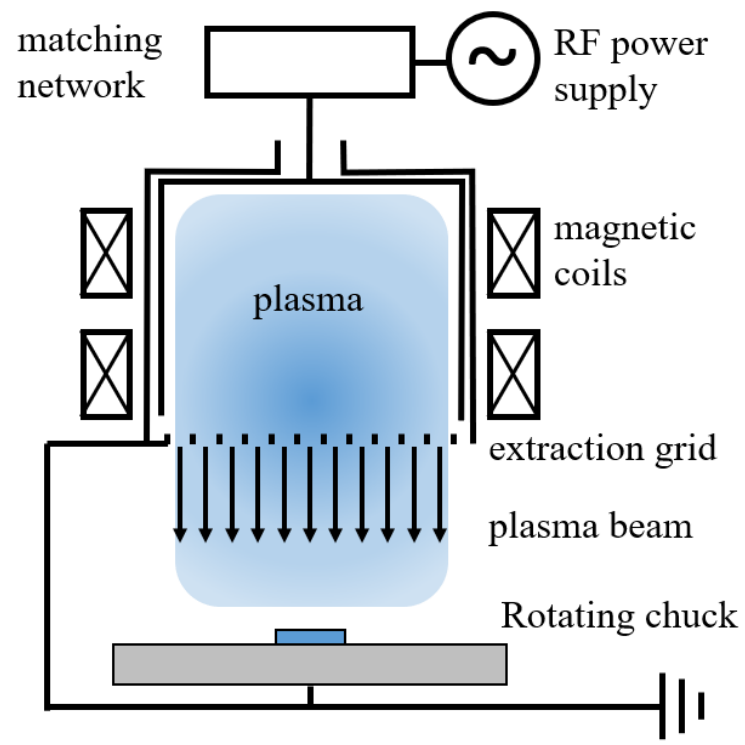

Figure 2: Schematic of the additional capacitive coupled RF-Plasma source (PSC) operating with argon and oxygen [4].

Transmission measurements of the deposited $\mathrm{Ta}_{2} \mathrm{O}_{5}$ thin films were performed using an Essent Photon RT spectrophotometer scanning the wavelength range from $250 \mathrm{~nm}$ to $980 \mathrm{~nm}$ in steps of $1 \mathrm{~nm}$. The layer uniformity was measured using a Sentech SENresearch ellipsometer, scanning the $200 \mathrm{~mm}$ diameter in steps of $5 \mathrm{~mm}$ with a $10 \mathrm{~mm}$ edge exclusion. The results were fitted using a Cauchy model for $\mathrm{Ta}_{2} \mathrm{O}_{5}$ on a silicon wafer. Roughness measurement involved using a Park NX20 atomic force microscope, scanning a $0.5 \mu \mathrm{m}$ square area with 516 pixels edge length. The density of the $\mathrm{Ta}_{2} \mathrm{O}_{5}$ layers has been measured with a Bruker D8 Discover XRD system.

An initial parameter study was performed in order to identify a process window for manufacturing $\mathrm{Ta}_{2} \mathrm{O}_{5}$ thin films showing low optical losses without making use of the PSC. This was then used as a starting point to find a working point where the PSC RF-plasma and the plasma from the magnetron system don't affect each other, and stable operation is possible. Using these settings, $455 \mathrm{~nm}$ thick $\mathrm{Ta}_{2} \mathrm{O}_{5}$ layers were produced at varying PSC power levels from $0 \mathrm{~kW}$ to $3 \mathrm{~kW}$ in steps of $500 \mathrm{~W}$. Three sets of the $\mathrm{Ta}_{2} \mathrm{O}_{5}$ layers were manufactured for this purpose. Thickness control was achieved using the abovedescribed GSM system to provide a precise turn-off.

\section{Measurements and results}

The spectral measurements of the three sets of $\mathrm{Ta}_{2} \mathrm{O}_{5}$ layers were used to evaluate the optical losses in the range from $380 \mathrm{~nm}$ to $450 \mathrm{~nm}$, calculated as follows:

$$
\text { Loss }=100 \%-\text { Transmission }- \text { Reflection }
$$

The resulting losses of the $\mathrm{Ta}_{2} \mathrm{O}_{5}$ layers can be seen in Figure 3 . With the exception of two coatings, the losses were found to be below the resolution of the spectrometer with no significant identifiable trend. The two $\mathrm{Ta}_{2} \mathrm{O}_{5}$ samples' higher losses were also slightly different behavior in the broader absorption spectrum compared to the other samples. The significantly higher loss values compared to the other samples can however be explained by the chosen evaluation wavelength range which is close to the $\mathrm{Ta}_{2} \mathrm{O}_{5}$ absorption edge [5]. All the coated layers can consequently be used for optical coating applications. For the purpose of further evaluations in this study, only set 2 was used.

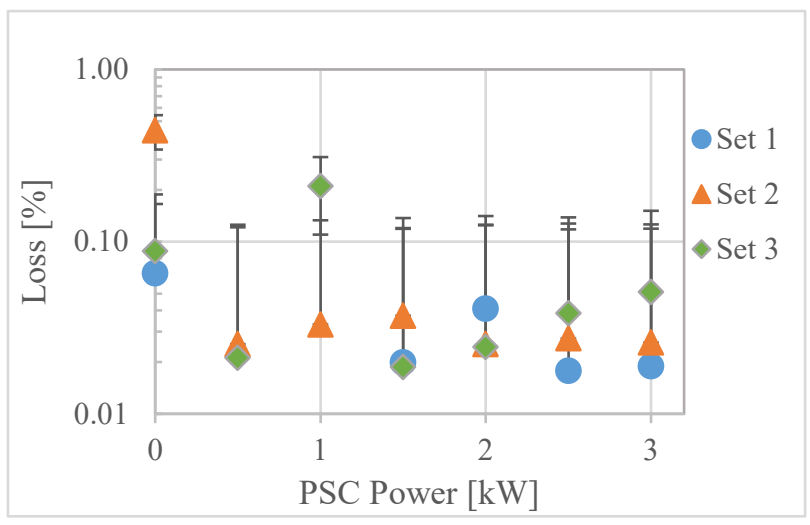

Figure 3: Calculated losses for the three sets of $\mathrm{Ta}_{2} \mathrm{O}_{5}$ layers.

The layer thickness measurements were normalized to $100 \%$ to eliminate any variations in thickness and then used to calculate the uniformity. For this purpose, the variation in thickness, $d_{v a r}$, was calculated from the maximum $\left(d_{\max }\right)$ and minimum $\left(d_{\min }\right)$ measured thicknesses as follows:

$$
d_{v a r}=\frac{d_{\max }-d_{\min }}{d_{\max }+d_{\min }}
$$

As shown in Figure 4, increasing the PSC power showed a positive effect on the uniformity distribution, reducing the thickness variation from $1.15 \%$ when not using the PSC to $0.31 \%$ when using $3 \mathrm{~kW}$ PSC power. To ensure the clarity of the results, the error bars for the uniformity plots have been omitted, but all lie in a close range between $0.19 \%$ to $0.21 \%$. The interpretation of the resulting uniformity improvement must, however, take into account that it usually just lowers the edge and raises the center; hence with a different starting distribution, the outcome can vary. In general, the table to substrate distance and used magnetic system will be the main influences on the uniformity [6]. 


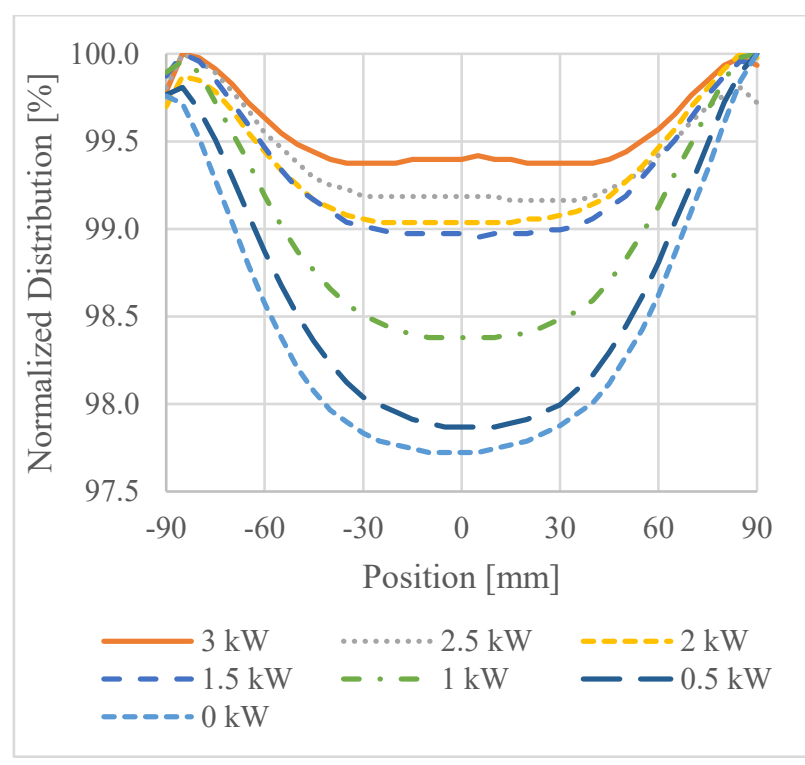

Figure 2: Normalized $\mathrm{Ta}_{2} \mathrm{O}_{5}$ layer uniformity for depositions performed at different PSC power levels.

The stress of the $\mathrm{Ta}_{2} \mathrm{O}_{5}$ layers was measured on single side polished silicon wafers before and after the coating process in order to take the substrate material variability into account. The compressive stress in the $\mathrm{Ta}_{2} \mathrm{O}_{5}$ layers was reduced with increased PSC power levels (Figure 5).

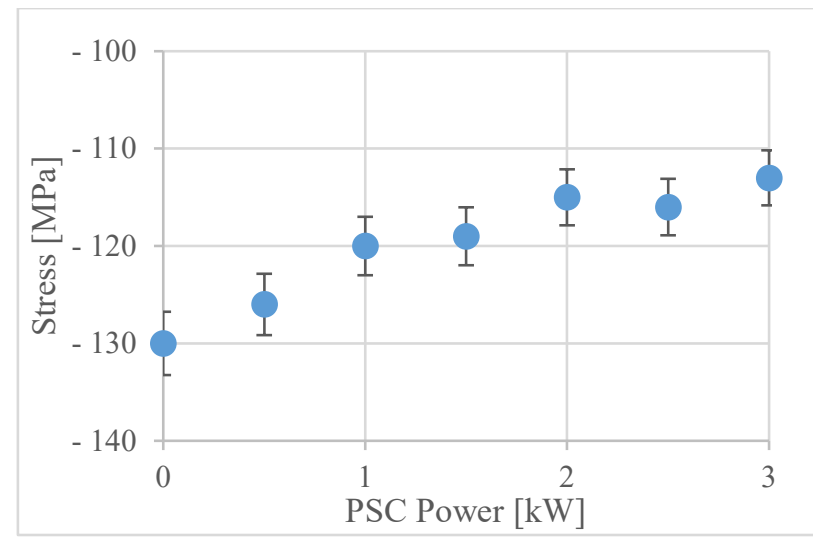

Figure 3: Stress in the $\mathrm{Ta}_{2} \mathrm{O}_{5}$ layers as a function of PSC power used during deposition.

An increase in PSC power during deposition was found to slightly reduce the roughness of the $\mathrm{Ta}_{2} \mathrm{O}_{5}$ layers, as can be seen in Figure 6. As all samples showed roughness levels in the lower Angstrom range, the measurement uncertainty is very large as the values are close to the detection limit of the AFM system.

Figure 7 shows the influence of the PSC power level on the refractive index of the $\mathrm{Ta}_{2} \mathrm{O}_{5}$ layers. In order to understand this, element analysis was performed to evaluate the oxygen and argon concentration in the films, which exhibited no trend (Figure 8).

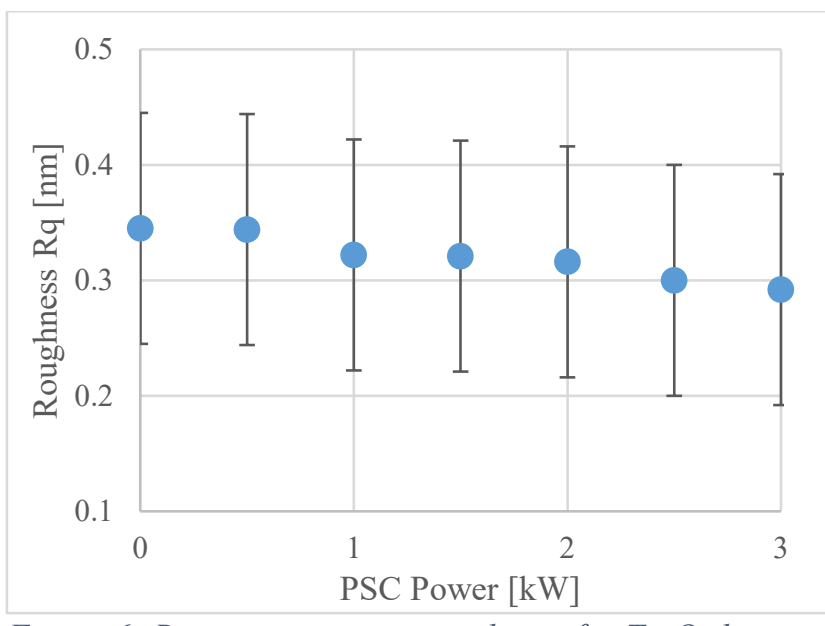

Figure 6: Root mean square roughness for $\mathrm{Ta}_{2} \mathrm{O}_{5}$ layers deposited at different PSC power levels.

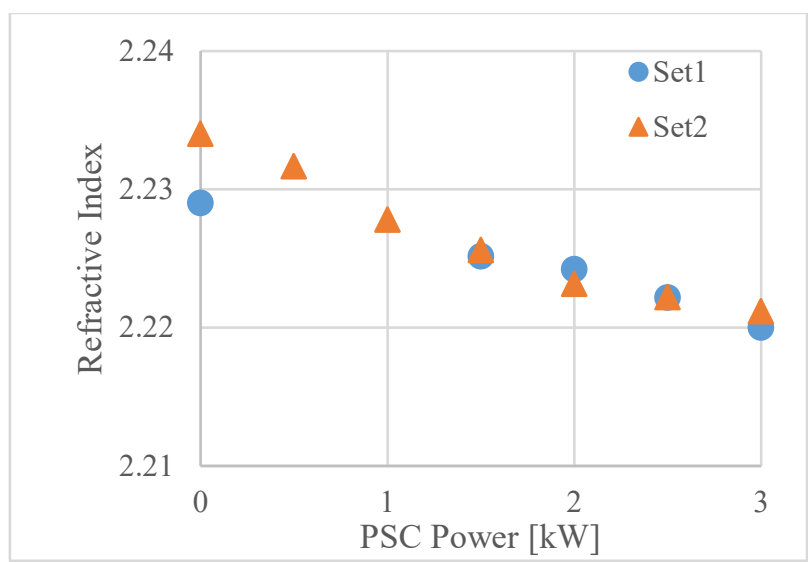

Figure 7: Observed change in refractive index with increasing PSC power during deposition.

Subsequent XRD measurements (Figure 9) showed a marked decrease in the density of the $\mathrm{Ta}_{2} \mathrm{O}_{5}$ layers when increasing the PSC power. A linear correlation could be observed when plotted against the refractive index, as shown in Figure 10.

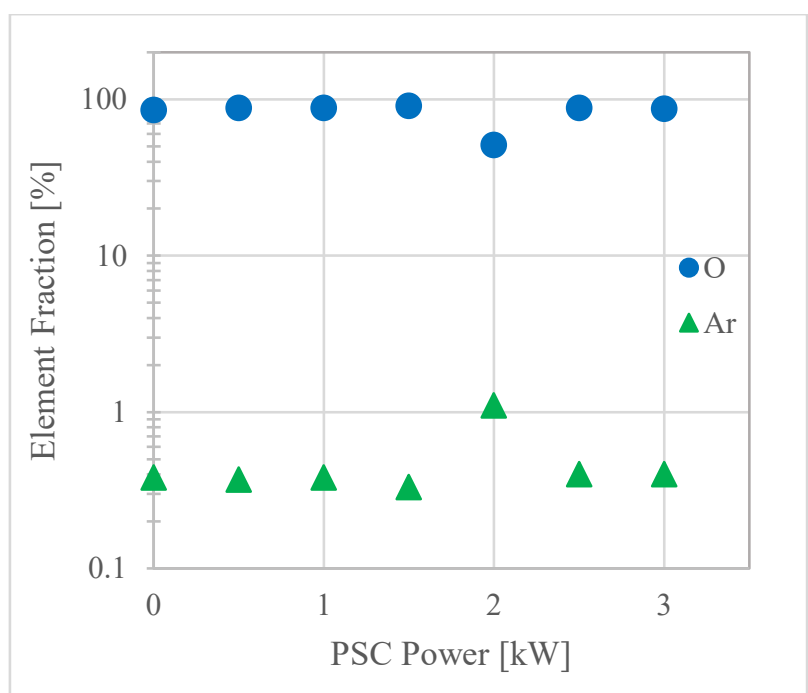

Figure 8: EDX element analysis of the $\mathrm{Ta}_{2} \mathrm{O}_{5}$ layers deposited at various PSC power levels. 


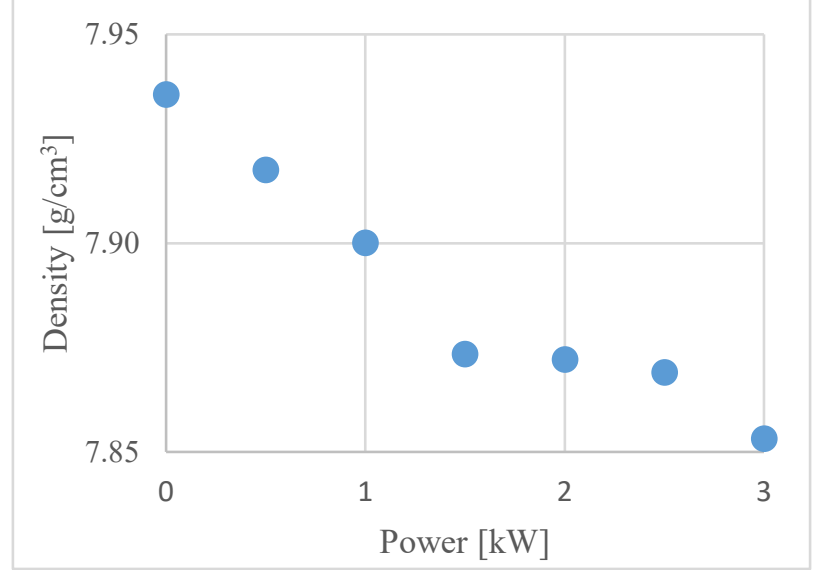

Figure 9: Density of the $\mathrm{Ta}_{2} \mathrm{O}_{5}$ layers as a function of increasing PSC power levels.

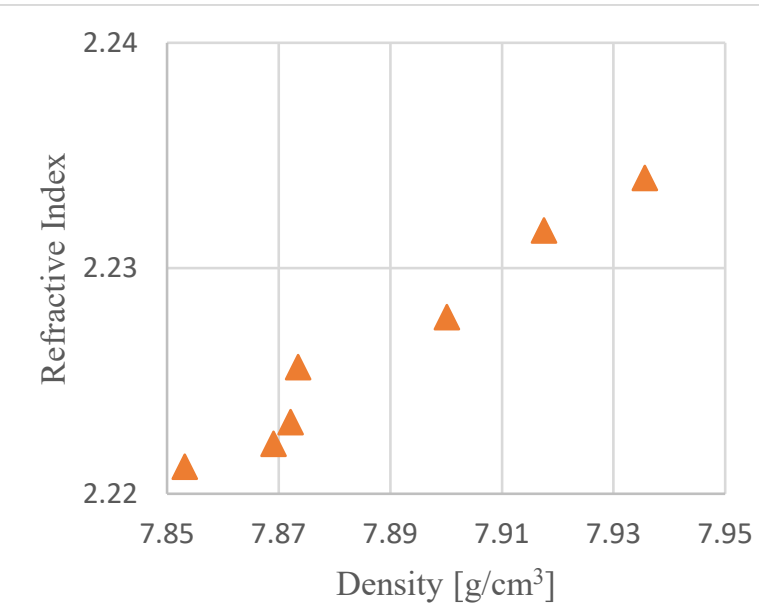

Figure 10: Refractive index as a function of the density of the $\mathrm{Ta}_{2} \mathrm{O}_{5}$ layers.

\section{Conclusion}

This study investigated the influence the PSC power level on the material properties of thin $\mathrm{Ta}_{2} \mathrm{O}_{5}$ layers. The strongest influence of the PSC was found to be on the stress in the thin $\mathrm{Ta}_{2} \mathrm{O}_{5}$ layers and the coating uniformity. The use of $3 \mathrm{~kW}$ power led to a decrease in the compressive stress in the $\mathrm{Ta}_{2} \mathrm{O}_{5}$ layers of approximately $10 \%$.The thickness variation over a $200 \mathrm{~mm}$ wafer substrate was found to reduce from $1.15 \%$ to $0.31 \%$ using the PSC. Although this improvement is not seen as a flattening of the deposition profile uniformity, it is seen as a lowering of the deposition thickness in the outer areas of the substrate and increased thickness in the center and the uniformity itself mainly depends on the table-substrate distance. This distance, however, also influences the plasma stability and therefore the possibility to use more stable distances and improve the resulting uniformity distribution can be helpful. A surprising result was the decrease in refractive index with increasing PSC power. Initial results of density measurements showed a linear correlation with the refractive index. Intuitively a layer from a similar material with lower density would result in a lower refractive index. It is, however, not clear why $\mathrm{Ta}_{2} \mathrm{O}_{5}$ layers coated with higher PSC power should decrease in density. This will be the subject of further investigations.

\section{Acknowledgment}

Special thanks to André Mocker and Jan Allaart from the OST University of Applied Science in Buchs SG, Switzerland, for assisting with the element analysis.

\section{Bibliography}

1. C. Chaneliere, J. L. Autran, R. A. B. Devine, B. Balland, Materials Science and Engeneering Reports, Tantalum pentoxide $\left(\mathrm{Ta}_{2} \mathrm{O}_{5}\right)$ thin films for advanced dielectric applications, Volume 22 Issue 6, (1998)

2. K. Chen, M, Nielsen, G. R. Yang, E. J. Rymaszewsky, T. -M. Lu, Journal of Electronic Materials, Study of Amorphous Ta2O5 thin films by DC magnetron reactive sputtering, Vol. 26, Nr.4 (1997)

3. J. E. Greene, Journal of Vacuum Science \& Technology, Tracing the recorded history of thin-film sputter deposition: From the 1800s to 2017, A 35, (2017)

4. S. Schwyn-Thöny, S. Gees, E. Schüngel, OSA Publishing, improving film stress and surface roughness by using a plasma source in magnetron sputtering, WC.4, (2019)

5. L. V. Rodríguez-de Marcos, J. I. Larruquert, J. A. Méndez, J. A. Aznárez, OSA Publishing, Selfconsistent optical constants of $\mathrm{SiO}_{2}$ and $\mathrm{Ta}_{2} \mathrm{O}_{5}$ films, Vol. 6 Issue 11, (2016)

6. M. Chesaux, S. Schwyn Thöny, S. Gees, A. Frigg, OSA Publishing, Rotating target Source: Novel shaperless concept for magnetron sputtering with excellent uniformity, WA.2, (2016) 